\title{
Surface Wave Scattering by an Elastic Plate Submerged in Water with Uneven Bottom
}

\section{Souvik Kundu and Rupanwita Gayen}

\author{
Department of Mathematics, Indian Institute of Technology, \\ 721302 Kharagpur, India \\ E-mail(corresp.): rupanwita.gayen@gmail.com \\ E-mail: kundusouvik@yahoo.co.in
}

Received May 13, 2019; revised February 28, 2020; accepted March 1, 2020

\begin{abstract}
Wave interaction with a vertical elastic plate in presence of undulating bottom topography is considered, assuming linear theory and utilizing simple perturbation analysis. First order correction to the velocity potential corresponding to the problem of scattering by a vertical elastic plate submerged in a fluid with a uniform bottom is obtained by invoking the Green's integral theorem in a suitable manner. With sinusoidal undulation at the bottom, the first-order transmission coefficient $\left(T_{1}\right)$ vanishes identically. Behaviour of the first order reflection coefficient $\left(R_{1}\right)$ depending on the plate length, ripple number, ripple amplitude and flexural rigidity of the plate is depicted graphically. Also, the resonant nature of the first order reflection is observed at a particular value of the ratio of surface wavelength to that of the bottom undulations. The net reflection coefficient due to the joint effect of the plate and the bottom undulation is also presented for different flexural rigidity of the plate. When the rigidity parameter is made sufficiently large, the results for $R_{1}$ reduce to the known results for a surface piercing rigid plate in water with bottom undulation.
\end{abstract}

Keywords: bottom undulation, vertical elastic plate, perturbation analysis, first-order reflection.

AMS Subject Classification: $76 \mathrm{~B} 15$.

\section{Introduction}

Waves traveling over a seabed with uniform finite depth experience no reflection when there is no obstacle. However, if surface waves are incident on a region with irregular bottom topography, the reflection and transmission phenomena of waves will take place. The problems of analysing wave motion in the presence of bottom deformation have gained considerable attention from

Copyright (c) 2020 The Author(s). Published by VGTU Press

This is an Open Access article distributed under the terms of the Creative Commons Attribution License (http://creativecommons.org/licenses/by/4.0/), which permits unrestricted use, distribution, and reproduction in any medium, provided the original author and source are credited. 
many researchers all over the world due to their importance in understanding the wave energy distribution near coastal regions. Some naturally occurring bottom obstacles are shore-parallel bars, sand ripples etc. which create obstructions to shoreline erosion by dissipating incident wave energy.

Although the exact forms/expressions of the reflection and transmission coefficients are difficult to obtain analytically, they can be estimated numerically. In relevant literature, we find that several mathematical techniques have been adopted to examine the effects of different bed-forms. One such method is based on the mapping of the actual fluid domain into some simpler ones, normally into a uniform strip (e.g. Kreisel [10], Roseau [25], Fitz-Gerald [7]). But such mappings are not convenient for arbitrary bottom variations. A numerical method based on integral equation formulation was applied by Newman [20], Miles [17] for simple topographic variation, such as step changes in depth. Davies [5] examined the problem of the interaction between surface waves and a patch of sinusoidal ripples in the bottom on the basis of linear perturbation theory followed by an application of Fourier transform technique. In his work, Davies [5] obtained the reflection and transmission coefficients for the 'far-field' and observed an oscillatory nature along with the resonance of the reflection. Later an extensive set of laboratory observations presented by Davies and Hearthershaw [6] confirmed the theoretical predictions made by Davies [5]. Staziker et al. [26] presented a mathematical method based on Green's function theory and a variational approach to study the problems of waves scattering by local bed elevation of arbitrary shapes.

There exists a class of problems dealing with small bottom deformations or slightly curved thin barriers which cannot be solved exactly but can be formulated by adding small correction terms to the mathematical solution of an exactly solvable problem. Perturbation analysis can be employed to deal with such problems. Perturbation analysis leads to an illustration in terms of a formal power series to get the required solution. We can see the use of perturbation technique in the work of several researchers. In the context of bottom undulations, Maiti and Mandal [12] investigated the scattering of incident waves in an ice-covered two-layer fluid. Banerjea et al. [1] used perturbation technique to study the effect of bottom undulation on the waves generated by an oscillating line source submerged beneath an ice-covered surface. Using regular perturbation analysis, Panda et al. [22] studied the flow problems in three layer fluid associated with small undulation at the bottom. Effect of the bottom undulations in a channel consisting of two immiscible fluids was considered by Mohapatra and Bora [19]. Later, Mohapatra [18] examined the effect of surface tension on the problem of wave scattering by bottom undulation in a two-layer fluid. Panda and Martha [21] considered a similar problem in presence of permeable bottom.

Apart from studying the sole effect of bottom undulation, it may be interesting as well as useful sometimes to investigate the joint effect of obstacles/surface discontinuity and bottom undulation. Since the bottom of an ocean is rarely of uniform depth throughout, it is worthwhile to study the effect of bottom undulation on wave-structure interaction problems for addressing realistic physical situations. Whenever uneven bottom is present, it causes wave transformation 
through the reflection of incoming waves and other phenomena like wave refraction and shoaling. Thus it is important to consider bottom undulations for modeling barriers. Again, the elastic plate has its own importance in constructing breakwaters due to longevity and cost-effectiveness. Also, the mathematical modelling becomes challenging when the propagating waves interact with the barrier as well as with the bottom undulations. These have motivated us to investigate the joint effect of the submerged vertical elastic plate and the bottom undulations. In literature, we can find some papers which study the combined effects of different types of obstacles and bottom undulations. Mandal and Gayen [14] employed perturbation analysis to investigate the problem of wave scattering by a surface-piercing barrier in presence of undulating bottom. Rakshit and Banerjea [24] used a similar perturbation technique to study the effect of small bottom undulation on the waves generated by rolling vertical plate either partially immersed or fully submerged in water. Again, Mandal and De [13] investigated the problem of wave scattering by bottom undulation in presence of a surface discontinuity. Choudhary and Martha [4] extended the work of Mandal and Gayen [14] for two different barrier configurations and included the graphs for hydrodynamic force and moment with respect to plate length.

In the context of linear water wave theory, wave interaction with submerged elastic plates is not a well studied topic. Meylan [16] investigated the problem of wave scattering by surface piercing vertical elastic plate to study the nature of the reflection and transmission. Peter and Meylan [23] used eigenfunction expansion to handle the time-dependent version of Meylan's [16] problem. Chakraborty et al. [2] presented a hypersingular integral equation technique to study the problem of wave scattering by a thin vertical elastic plate. Kundu et al. [11] used the method of Chakraborty et al. [2] to investigate the scattering problem in presence of an elastic plate with arbitrary inclinations.

In the present paper, we employ the aforesaid perturbation method to study the problem of wave scattering by a submerged vertical elastic plate in presence of undulating bottom of the domain. Application of the perturbation method produces boundary value problems (BVPs) for the potential functions of order zero and one respectively. The first problem (henceforth denoted as BVP-0) is related to the problem of wave scattering by a vertical elastic plate in uniform finite depth. The second boundary value problem $(B V P-1)$ represents the radiation problem in water of uniform finite depth and involves the solution of $B V P-0$ in its bottom condition. Without solving $B V P-1$ explicitly, using Green's integral theorem the first order reflection and transmission coefficients can be represented in terms of the shape function (describing the bottom undulation) and the solution of $B V P-0$. The expression of the first order reflection coefficient $\left(R_{1}\right)$ involves a particular form of the normal derivative of the zeroth order potential function. To find that we use a predefined mathematical technique based on the applications of the Green's function, Green's integral theorem and an expansion-collocation method (cf. Chakraborty et al. [2]). On the other hand, the first order transmission coefficient $\left(T_{1}\right)$ vanishes identically with the assumption of symmetry of sinusoidal undulations at the bottom. Finally, $\left|R_{1}\right|$ is depicted graphically for several values of the flexural rigidity, 
barrier depth, ripples' amplitude and ripple numbers. From these results, and from the depicted values of the first-order corrections to the zeroth order reflection coefficient $\left(R_{0}+\epsilon R_{1}\right)$ with respect to the dimensionless wave number, one can understand the joint effect of a vertical elastic plate and the undulating bottom topography on the propagation of surface waves. Graphical representation of $\left|R_{1}\right|$ with respect to the ratio of incident wavelength and the wavelength associated with bottom undulation is given. This gives a clear idea about the resonant nature of the first order reflection coefficient near a particular value of the above mentioned ratio. The results for $\left|R_{1}\right|$ are verified in two ways, one by comparing the known results for a surface piercing rigid plate to those for an elastic plate with sufficiently large rigidity; another by comparing the existing results for the sole effect of bottom undulations to those for a negligible length of the elastic plate.

\section{Mathematical formulation}

A two-dimensional potential flow is considered in the water domain of finite depth. In the corresponding Cartesian coordinate system $y=0$ represents the undisturbed free surface and the positive $y$-axis is taken vertically downwards (see Figure 1). The bottom of the domain has a small undulation which is described by $y=h+\epsilon c(x)$. Here the continuous and bounded function $c(x)$ indicates the shape of the undulation and is thus non-zero within the interval of $x$-axis where the undulation is present. Beyond this interval, $c(x)$ is identically equal to zero. The dimensionless parameter $\epsilon(\ll 1)$ represents the smallness of the irregularity at the bottom of the water domain. The vertical elastic plate occupies the region $x=0, l_{1}<y<l_{2}$. It is assumed that the motion is

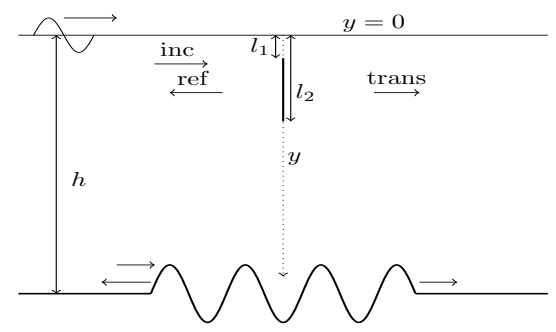

Figure 1. Physical representation of the problem.

irrotational, time harmonic and the fluid is inviscid and incompressible. Again, considering the linear water wave theory the velocity potential can be expressed as $\operatorname{Re}\left\{\phi(x, y) \mathrm{e}^{-\mathrm{i} \sigma t}\right\}$ (cf. [3]) where $\sigma$ is the angular frequency. Then the complex valued potential function $\phi(x, y)$ satisfies

$$
\begin{aligned}
& \frac{\partial^{2} \phi}{\partial x^{2}}+\frac{\partial^{2} \phi}{\partial y^{2}}=0 \text { in the fluid region, } \\
& K \phi+\phi_{y}=0 \text { on } y=0,-\infty<x<\infty
\end{aligned}
$$


with $K=\sigma^{2} / g(g$ is acceleration due to gravity $)$,

$$
\frac{\partial \phi}{\partial n}=0 \text { on } y=h+\epsilon c(x)
$$

$n$ denoting the normal direction at any point of the undulating bottom. We should also specify the edge condition

$$
\nabla \phi \sim O\left(r^{-1 / 2}\right) \text { as } r \rightarrow 0
$$

$r$ indicating the distance of an arbitrary point in the water region from either end of the elastic plate and the radiation conditions

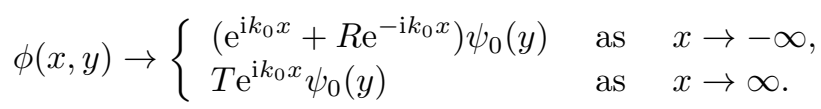

Here $\psi_{0}(y)=N_{0}^{-1} \cosh k_{0}(h-y), N_{0}=\left[\left(2 k_{0} h+\sinh 2 k_{0} h\right) /\left(4 k_{0} h\right)\right]^{1 / 2}$ and $k_{0}$ is the real positive root of $k \tanh k h=K$. In equation (2.5), $R$ and $T$ represent the reflection and transmission coefficients respectively.

The condition on the elastic plate is given by (cf. [16])

$$
D \frac{\mathrm{d}^{4} \phi_{x}}{\mathrm{~d} y^{4}}-m K \phi_{x}=K\left(\phi^{-}-\phi^{+}\right) \text {on } x=0, l_{1}<y<l_{2} .
$$

Here $\phi^{-}=\phi\left(0^{-}, y\right)$ and $\phi^{+}=\phi\left(0^{+}, y\right)$ denote the potentials on the left and the right sides of the plate. $D=\frac{E h^{\prime 3}}{12 \rho\left(1-\nu^{2}\right) g}$ is the stiffness of the elastic plate, $E$ represents the Young's modulus, $\nu$ denotes the Poisson's ratio. Again, $m=\rho^{\prime} / \rho h^{\prime}, \rho$ and $\rho^{\prime}$ are the densities of water and plate material, $h^{\prime}$ is its negligible thickness. Also, when the top end of the plate is clamped and the bottom end is free then the end conditions are given by

$$
\frac{\mathrm{d} \phi_{x}}{\mathrm{~d} y}=0=\phi_{x} \text { at } y=l_{1} \text { and } \frac{\mathrm{d}^{2} \phi_{x}}{\mathrm{~d} y^{2}}=0=\frac{\mathrm{d}^{3} \phi_{x}}{\mathrm{~d} y^{3}} \text { at } y=l_{2} .
$$

\section{Method of solution}

To implement the solution procedure as described in Section 1 we observe that the bottom condition (2.3) can be expressed as (cf. [14])

$$
\frac{\partial \phi}{\partial y}-\epsilon \frac{\mathrm{d}}{\mathrm{d} x}\left\{c(x) \frac{\partial \phi(x, h)}{\partial x}\right\}+O\left(\epsilon^{2}\right)=0 \text { on } y=h
$$

The form of the above approximation (3.1) and the smallness of the parameter $\epsilon$ indicate that the potential function and the constants $R$ and $T$ can be expanded as

$$
\begin{aligned}
& \phi(x, y ; \epsilon)=\phi_{0}+\epsilon \phi_{1}+O\left(\epsilon^{2}\right), \quad R(\epsilon)=R_{0}+\epsilon R_{1}+O\left(\epsilon^{2}\right), \\
& T(\epsilon)=T_{0}+\epsilon T_{1}+O\left(\epsilon^{2}\right) .
\end{aligned}
$$


Substituting the above expansions in (2.1)-(2.2), (2.4)-(2.6), (3.1) and equating the coefficients of $\epsilon$ up to first order terms we find that $\phi_{0}$ and $\phi_{1}$ satisfy two boundary value problems namely $B V P-0$ for $\phi_{0}$ and $B V P-1$ for $\phi_{1}$.

$B V P-0$ (for the function $\left.\phi_{0}(x, y)\right)$ :

$$
\begin{aligned}
& \frac{\partial^{2} \phi_{0}}{\partial x^{2}}+\frac{\partial^{2} \phi_{0}}{\partial y^{2}}=0 \text { in } 0<y<h,-\infty<x<\infty, \\
& K \phi_{0}+\frac{\partial \phi_{0}}{\partial y}=0 \text { on } y=0,-\infty<x<\infty, \\
& \frac{\partial \phi_{0}}{\partial y}=0 \text { on } y=h, \\
& D \frac{\mathrm{d}^{4} \phi_{0 x}}{\mathrm{~d} y^{4}}-m K \phi_{0 x}=K\left(\phi_{0}^{-}-\phi_{0}^{+}\right) \text {on } x=0, l_{1}<y<l_{2}, \\
& \nabla \phi_{0} \sim O\left(r^{-1 / 2}\right) \text { as } r \rightarrow 0, \\
& \phi_{0}(x, y) \rightarrow \begin{cases}\left(\mathrm{e}^{\mathrm{i} k_{0} x}+R_{0} \mathrm{e}^{-\mathrm{i} k_{0} x}\right) \psi_{0}(y) \text { as } & x \rightarrow-\infty, \\
T_{0} \mathrm{e}^{\mathrm{i} k_{0} x} \psi_{0}(y) & \text { as } x \rightarrow \infty .\end{cases}
\end{aligned}
$$

$B V P-1$ (for the function $\left.\phi_{1}(x, y)\right)$ :

$$
\begin{aligned}
& \frac{\partial^{2} \phi_{1}}{\partial x^{2}}+\frac{\partial^{2} \phi_{1}}{\partial y^{2}}=0 \text { in } 0<y<h,-\infty<x<\infty \\
& K \phi_{1}+\frac{\partial \phi_{1}}{\partial y}=0 \text { on } y=0,-\infty<x<\infty \\
& \frac{\partial \phi_{1}}{\partial y}=\frac{\mathrm{d}}{\mathrm{d} x}\left\{c(x) \phi_{0 x}\right\} \text { on } y=h \\
& D \frac{\mathrm{d}^{4} \phi_{1 x}}{\mathrm{~d} y^{4}}-m K \phi_{1 x}=K\left(\phi_{1}^{-}-\phi_{1}^{+}\right) \text {on } x=0, l_{1}<y<l_{2},
\end{aligned}
$$

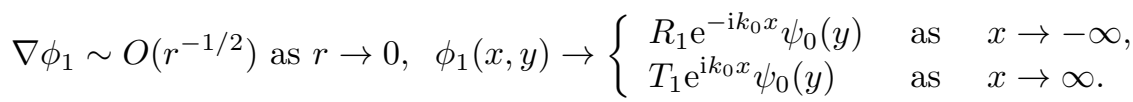

$$
\begin{aligned}
& \underset{(-X, h)}{(X, X)}(X=0
\end{aligned}
$$

Figure 2. Contour for application of Green's integral theorem.

The solution of $B V P-1$ depends on the solution of $B V P-0$. It is interesting to note that the values of $R_{1}$ and $T_{1}$ can be found without solving for $\phi_{1}$ and simply using integrals involving $c(x)$ and $\phi_{0 x}$ as determined in equations (3.4) and (3.5). This can be achieved by applying Green's integral theorem on the 
potential functions $\phi_{0}(x, y)$ and $\phi_{1}(x, y)$ over the region bounded externally by the lines

$$
y=0,-X \leq x \leq X ; x=-X, 0 \leq y \leq h ; y=h,-X \leq x \leq X ; x=X, 0 \leq y \leq h
$$

and internally by a contour enclosing the submerged elastic plate (see Figure 2).

Considering the limit $X \rightarrow \infty$ and shrinking the contour enclosing the plate we determine $R_{1}$ as

$$
2 \mathrm{i} k_{0} R_{1} h=\int_{-\infty}^{\infty} c(x) \phi_{0 x}^{2}(x, h) \mathrm{d} x .
$$

Again applying Green's integral theorem on $\phi_{0}(-x, y)$ and $\phi_{1}(x, y)$ in the same region we obtain

$$
2 \mathrm{i} k_{0} T_{1} h=\int_{-\infty}^{\infty} c(x) \phi_{0 x}(x, h) \phi_{0 x}(-x, h) \mathrm{d} x .
$$

Before we proceed further we must mention that our analysis is valid for small bottom deformations such as the presence of natural sand ripples at the bottom. The ripples have similar forms as that of sinusoidal functions. We thus consider $c(x)$ as

$$
c(x) \rightarrow \begin{cases}c_{0} \sin \lambda x, & -\frac{M \pi}{\lambda} \leq x \leq \frac{M \pi}{\lambda} \\ 0, & \text { otherwise }\end{cases}
$$

Here $M$ is a positive integer denoting the number of ripples at the bottom, $\lambda$ is the wave number of each ripple and $c_{0}$ represents the ripple amplitude.

Substituting the above form of $c(x)$ into equation (3.5) we find that $T_{1}$ vanishes identically where we have used the condition $c(-x)=-c(x)$. Now, equation (3.4) can be manipulated as

$$
2 \mathrm{i} k_{0} R_{1} h=\int_{0}^{\infty} c(x)\left\{\phi_{0 x}(x, h)-\phi_{0 x}(-x, h)\right\}\left\{\phi_{0 x}(x, h)+\phi_{0 x}(-x, h)\right\} \mathrm{d} x .
$$

From the above expression $R_{1}$ can be estimated numerically if we can compute the values of $\phi_{0 x}(x, h)$ and $\phi_{0 x}(-x, h)$. For finding the values of $\phi_{0 x}(x, h)$ and $\phi_{0 x}(-x, h)$ we solve equation (3.3) using the end conditions

$$
\frac{\mathrm{d} \phi_{0 x}}{\mathrm{~d} y}=0=\phi_{0 x} \text { at } y=l_{1} \text { and } \frac{\mathrm{d}^{2} \phi_{0 x}}{\mathrm{~d} y^{2}}=0=\frac{\mathrm{d}^{3} \phi_{0 x}}{\mathrm{~d} y^{3}} \text { at } y=l_{2} .
$$

It may be noticed that the solution $\phi_{0 x}$ to the differential equation (3.3) involves the unknown potential difference across the elastic plate. This unknown function is determined numerically by solving a hypersingular integral equation and using the solution, finally the normal derivative of $\phi_{0}$ on the plate is calculated (cf. [11]). The method is briefly illustrated in Appendix. 


\section{Numerical results}

In this section, we present some relevant graphs of absolute values of $R_{1}$ and $R_{0}+\epsilon R_{1}$ depending on the changes of different parametric values. Physical quantities are made dimensionless with respect to the uniform depth of the water domain. The plate is clamped at the upper end and free at the lower end.

In Figure 3, we present the values of $\left|R_{1}\right|$ as a function of $K h$ to compare the results for a rigid plate (cf. [14]) to that of the elastic plate. We consider a negligible submergence depth $\left(l_{1} / h=0.001\right)$ of the upper end of the plate to compare our results with those for a partially immersed barrier. It is clear that when $D$ is large enough $\left[D / h^{4}=10\right]$, the absolute values of the first order reflection coefficient for an elastic plate completely agree with those for a rigid plate.

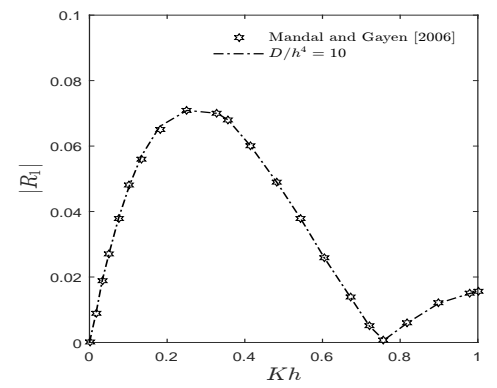

Figure 3. Comparison of the magnitudes of $\left|R_{1}\right|$ between rigid and elastic plates with

$D / h^{4}=10, l_{1} / h=0.001, l_{2} / h=$

$0.1, M=1, c_{0} / h=0.1, \lambda h=1$ and $m / h=0.001$.

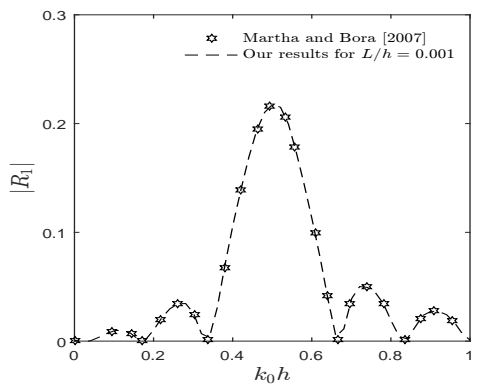

Figure 4. Comparison of the magnitudes of $\left|R_{1}\right|$ between the results for the sole effect of bottom undulation [15] and our results with negligible plate lenth for fixed $M=3, c_{0} / h=0.1, \lambda h=1$ and $l_{1} / h=0.1$.

Figure 4 represents the fact that the values of $\left|R_{1}\right|$ are the same in both the cases when there is no plate (cf. [15]) and when the dimensionless plate length $L / h=\frac{l_{2}-l_{1}}{h}$ is very small. Values of other parameters are kept fixed as $M=3$, $c_{0} / h=0.1, D / h^{4}=1, \lambda h=1$ and $m / h=0.001$. These two figures give a check on the correctness of our numerical results.

Figure 5 depicts the values of $\left|R_{1}\right|$ with respect to $K h$ for different values of $D / h^{4}$, whereas Figure 6 represents the values of $\left|R_{0}+\epsilon R_{1}\right|$ as a function of $K h$. In these two figures the rest of the parametric values are fixed as $l_{1} / h=0.1, l_{2} / h=0.2, M=1, c_{0} / h=0.1, \lambda h=1$ and $m / h=0.001$. For Figure 6 we consider $\epsilon=0.001$. These figures illustrate the fact that the values of $\left|R_{1}\right|$ as well as the values of $\left|R_{0}+\epsilon R_{1}\right|$ are increasing with increasing values of stiffness $\left(D / h^{4}\right)$. As $D$ becomes sufficiently large i.e. when $D / h^{4} \geq 1$, the elastic plate behaves as a rigid plate. After a certain level $\left(D / h^{4}=1\right)$ the changes are not significant. Also, if we compare the corresponding curves of Figure 5 and Figure 6, we observe that the presence of the elastic plate increases 


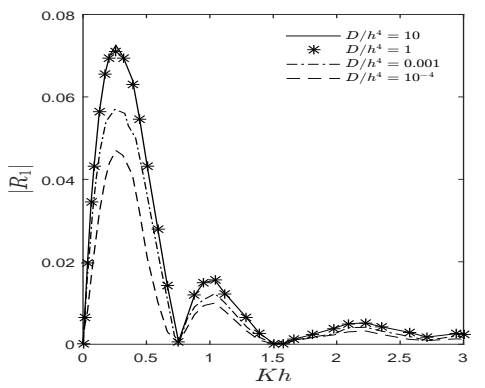

Figure 5. Variation of the values of $\left|R_{1}\right|$ with respect to $K h$ for different values of $D / h^{4}$ when $l_{1} / h=0.1, l_{2} / h=$ $0.2, M=1, c_{0} / h=0.1, \lambda h=1$ and $m / h=0.001$.

the reflection to some extent.

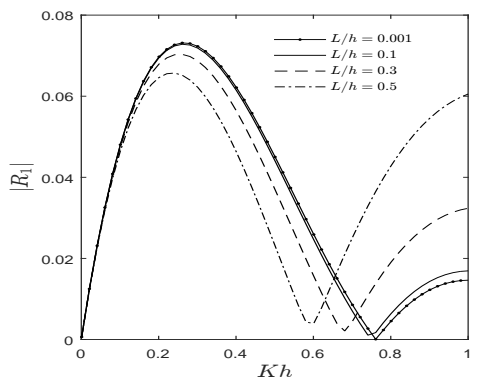

Figure 7. $\left|R_{1}\right|$ for different plate lengths with $M=1, c_{0} / h=0.1, l_{1} / h=$ $0.01, D / h^{4}=1$.

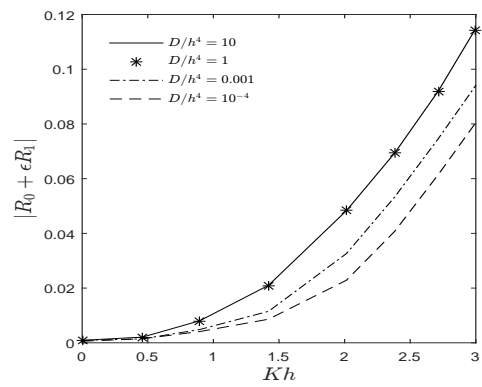

Figure 6. Variation of the values of $\left|R_{0}+\epsilon R_{1}\right|$ with respect to $K h$ for different values of $D / h^{4}$ when $l_{1} / h=$ $0.1, l_{2} / h=0.2, M=1, c_{0} / h=0.1, \lambda h=1$ and $m / h=0.001$.

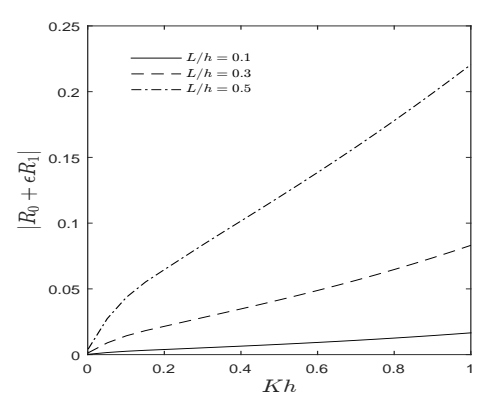

Figure 8. $\left|R_{0}+\epsilon R_{1}\right|$ for different plate lengths with $M=1, c_{0} / h=$ $0.1, l_{1} / h=0.01, D / h^{4}=1$.

Figure 7 represents the changes in the nature of the graph of absolute value of the first order reflection coefficient $\left(\left|R_{1}\right|\right)$ with respect to non-dimensional wave number $(K h)$ for various values of the non-dimensional plate length $(L / h=0.001,0.1,0.3,0.5)$ when the value of $l_{1} / h(=0.01)$ is fixed. Here, the number of ripples and the ripple amplitude are also kept fixed $\left(M=1, c_{0} / h=\right.$ 0.1 . From this figure, we realize the fact that $\left|R_{1}\right|$ has an oscillatory nature as a function of $K h$ and the zeros of $\left|R_{1}\right|$ are shifted towards the left with an increasing plate length. It indicates that with increasing plate length the zero values of $\left|R_{1}\right|$ appear at some lower frequency of the incoming wave. In other words, we can say at those particular frequencies the contribution of the bottom undulation in reflecting incoming waves is negligible. In Figure 8, the absolute values of $R_{0}+\epsilon R_{1}$ are plotted for different plate lengths $L / h=0.1,0.3,0.5$. Other parametric values are kept fixed as $l_{1} / h=0.01, M=1, c_{0} / h=0.1$. Through Figures 7 and 8, it can be noticed that with increasing plate length the peak value of $\left|R_{1}\right|$ decreases whereas the value of $\left|R_{0}+\epsilon R_{1}\right|$ increases 
significantly.

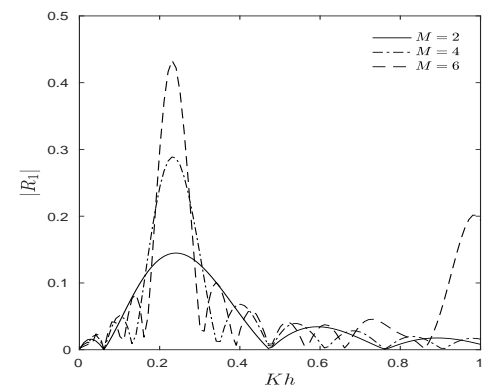

Figure 9. $\left|R_{1}\right|$ for different ripple number with

$l_{1} / h=0.05, l_{2} / h=0.2, c_{0} / h=0.1$.

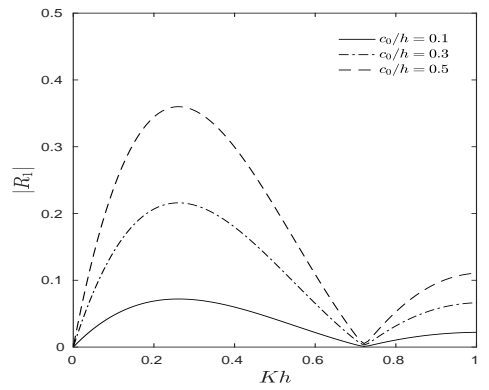

Figure 10. $\left|R_{1}\right|$ for different ripple amplitude with $l_{1} / h=0.01, l_{2} / h=0.2, M=1$.

Figure 9 shows the effect of ripple numbers on $\left|R_{1}\right|$ for the fixed values of $l_{1} / h=0.05, l_{2} / h=0.2$ and $c_{0} / h=0.1$. The value of $\left|R_{1}\right|$ increases and becomes more oscillatory with increasing number of ripples. The reason behind this outcome is the multiple interactions of the incident wave with ripple tops. In Figure 10, the absolute value of the first order reflection coefficient is depicted against $K h$ for various ripple amplitude. It is clear that for higher ripple amplitudes $\left|R_{1}\right|$ increases. In this figure, the fixed values are $l_{1} / h=0.01, l_{2} / h=$ 0.2 and $M=1$.

Bragg reflection over varying topography is an important topic to discuss in the context of problems dealing with bottom irregularities. Several researchers have studied the developed models of Bragg reflection (cf. Kirby [9]). Through these studies, ocean engineers can make a clear idea about the peak value of reflection due to bottom undulation and the condition of its occurrence. In early studies, it has been demonstrated that in a simple case of a patch of sinusoidal ripples in the bottom, the resonant nature of the first order reflection depends on the wavelengths of the incoming wave and that of the undulating bottom.

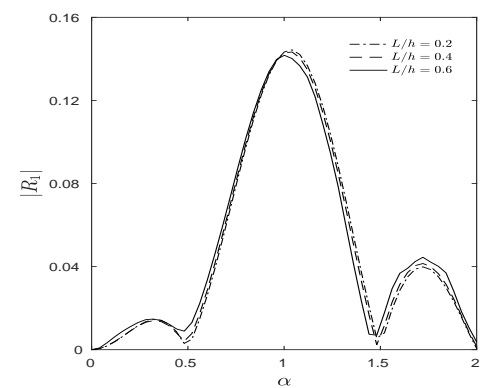

Figure 11. $\left|R_{1}\right|$ versus $\alpha$ for different values of plate length with $l_{1} / h=$ $0.01, c_{0} / h=0.1, M=2, K h=0.25$.

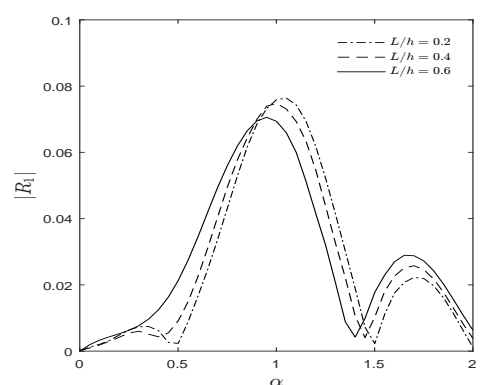

Figure 12. $\left|R_{1}\right|$ versus $\alpha$ for different values of plate length with $l_{1} / h=$ $0.01, c_{0} / h=0.1, M=2, K h=1.5$.

In Figures 11 and 12 we represent the graphs of $\left|R_{1}\right|$ with respect to $\alpha\left(=\frac{2 k_{0}}{\lambda}\right)$ 
for various values of $L / h$ with fixed values of $l_{1}=0.01, M=2, c_{0} / h=0.1$ and $D / h^{4}=1$. In Figure $11, K h=0.25$ whereas in Figure $12, K h=1.5$. The value of $\alpha$ for which $\left|R_{1}\right|$ attains its highest value is almost unity. Though in Figure 11, the differences between the peak values of $\left|R_{1}\right|$ for different values of $L / h$ are not clearly visible but for a moderate value of $K h(=1.5)$ it is detectable that with increasing plate length the peak values of $\left|R_{1}\right|$ are shifted to the left.

\section{Conclusions}

The problem of wave scattering by a thin elastic plate in the presence of an undulating bottom has been studied. Employing a simplified perturbation analysis the first-order reflection and transmission coefficients are expressed in terms of integrals involving the shape function describing the bottom deformation and the normal derivative of potential function related to the problem of uniform finite depth. A particular case of bottom deformation has been considered in the form of sinusoidal ripples. For this particular choice, the first order transmission coefficient vanishes and the first order reflection coefficient is calculated numerically and presented graphically for different parametric values. Due to multiple interactions of waves with an elastic plate and ripples at the bottom, $\left|R_{1}\right|$ shows an oscillatory nature. From the graphs presented above, it is quite clear that the value of $\left|R_{1}\right|$ increases in absence of a barrier (see Figure 7 ). It has been established through graphical comparison with published results (see Figure 1) that the values of $\left|R_{1}\right|$ in the presence of an elastic plate for large rigidity are the same as those in the presence of a rigid plate (cf. Mandal and Gayen [14]). The variations of $\left|R_{1}\right|$ and $\left|R_{0}+\epsilon R_{1}\right|$ with varying flexural rigidity of the plate show that the net reflection increases as the plate tends to become rigid. Comparison of graphs for $\left|R_{0}+\epsilon R_{1}\right|$ with those for $\left|R_{1}\right|$ clarify the fact that the joint effect of the plate and the small bottom undulation increases the reflection due to the sole effect of bottom undulation to some extent. $\left|R_{1}\right|$ has also been presented with respect to the parametric value $\alpha$ denoting the ratio of the wavelength of the incoming wave to that of the bottom undulation, from which an observation can be made about the resonant interactions of waves with the ripples. These observations might be useful in modelling a flexible breakwater placed in a fluid region with small bottom undulations.

\section{References}

[1] S. Banerjea, P. Rakshit and P. Maiti. On the waves generated due to a line source present in an ocean with an ice cover and a small bottom undulation. Fluid Dynamics Research, 43(2):025506, 2011. https://doi.org/10.1088/0169$5983 / 43 / 2 / 025506$.

[2] R. Chakraborty, A. Mondal and R. Gayen. Interaction of surface water waves with a vertical elastic plate: a hypersingular integral equation approach. Zeitschrift für angewandte Mathematik und Physik, 67(5):115, 2016. https://doi.org/10.1007/s00033-016-0709-0. 
[3] P.G. Chamberlain and D. Porter. The modified mild-slope equation. Journal of Fluid Mechanics, 291:393-407, 1995. https://doi.org/10.1017/S0022112095002758.

[4] A. Choudhary and S.C. Martha. Diffraction of surface water waves by an undulating bed topography in the presence of vertical barrier. Ocean Engineering, 122:32-43, 2016. https://doi.org/10.1016/j.oceaneng.2016.06.013.

[5] A.G. Davies. The reflection of wave energy by undulations on the seabed. Dynamics of Atmospheres and Oceans, 6(4):207-232, 1982. https://doi.org/10.1016/0377-0265(82)90029-X.

[6] A.G. Davies and A.D. Heathershaw. Surface-wave propagation over sinusoidally varying topography. Journal of Fluid Mechanics, 144:419-443, 1984. https://doi.org/10.1017/S0022112084001671.

[7] G.F. Fitz-Gerald. The reflexion of plane gravity waves travelling in water of variable depth. Philosophical Transactions of the Royal Society of London. Series A, Mathematical and Physical Sciences, 284(1317):49-89, 1976. https://doi.org/10.1098/rsta.1976.0099.

[8] R. Gayen and A. Mondal. Water wave interaction with two symmetric inclined permeable plates. Ocean Engineering, 124:180-191, 2016. https://doi.org/10.1016/j.oceaneng.2016.07.045.

[9] J.T. Kirby. A note on Bragg scattering of surface waves by sinusoidal bars. Physics of Fluids A: Fluid Dynamics, 5(2):380-386, 1993. https://doi.org/10.1063/1.858861.

[10] G. Kreisel. Surface waves. Quarterly of Applied Mathematics, 7(1):21-44, 1949. https://doi.org/10.1090/qam/31924.

[11] S. Kundu, R. Gayen and R. Datta. Scattering of water waves by an inclined elastic plate in deep water. Ocean Engineering, 167:221-228, 2018. https://doi.org/10.1016/j.oceaneng.2018.07.054.

[12] P. Maiti and B.N. Mandal. Water wave scattering by bottom undulations in an ice-covered two-layer fluid. Applied Ocean Research, 30(4):264-272, 2008. https://doi.org/10.1016/j.apor.2008.11.004.

[13] B.N. Mandal and S. De. Surface wave propagation over small undulations at the bottom of an ocean with surface discontinuity. Geophysical $\&$ Astrophysical Fluid Dynamics, 103(1):19-30, 2009. https://doi.org/10.1080/03091920802390073.

[14] B.N. Mandal and R. Gayen. Water wave scattering by bottom undulations in the presence of a thin partially immersed barrier. Applied Ocean Research, 28(2):113-119, 2006. https://doi.org/10.1016/j.apor.2006.06.002.

[15] S.C. Martha and S.N. Bora. Oblique surface wave propagation over a small undulation on the bottom of an ocean. Geophysical \& Astrophysical Fluid Dynamics, 101(2):65-80, 2007. https://doi.org/10.1080/03091920701208186.

[16] M.H. Meylan. A flexible vertical sheet in waves. The International Journal of Offshore and Polar Engineering, 5(02):105-110, 1994. Available from Internet: https://www . onepetro.org/conference-paper/ISOPE-I-94-150.

[17] J.W. Miles. Surface-wave scattering matrix for a shelf. Journal of Fluid Mechanics, 28(4):755-767, 1967. https://doi.org/10.1017/S0022112067002423.

[18] S. Mohapatra. The effect of free-surface tension on scattering of water waves by small bottom undulation. ANZIAM Journal, 58(0):39-80, 2017. https://doi.org/10.21914/anziamj.v58i0.11232. 
[19] S. Mohapatra and S.N. Bora. Reflection and transmission of water waves in a two-layer fluid flowing through a channel with undulating bed. Zeitschrift für Angewandte Mathematik und Mechanik, 91(1):46-56, 2011. https://doi.org/10.1002/zamm.200800216.

[20] J.N. Newman. Propagation of water waves over an infinite step. Journal of Fluid Mechanics, 23(2):399-415, 1965. https://doi.org/10.1017/S0022112065001453.

[21] S. Panda and S.C. Martha. Water-waves scattering by permeable bottom in twolayer fluid in the presence of surface tension. Mathematical Modelling $\&$ Analysis, 22(6):827-851, 2017. https://doi.org/10.3846/13926292.2017.1386239.

[22] S. Panda, S.C. Martha and A. Chakrabarti. Three-layer fluid flow over a small obstruction on the bottom of a channel. The ANZIAM Journal, 56(3):248-274, 2015. https://doi.org/10.1017/S1446181114000480.

[23] M.A. Peter and M.H. Meylan. A general spectral approach to the timedomain evolution of linear water waves impacting on a vertical elastic plate. SIAM Journal on Applied Mathematics, 70(7):2308-2328, 2010. https://doi.org/10.1137/090756557.

[24] P. Rakshit and S. Banerjea. Effect of bottom undulation on the waves generated due to rolling of a plate. Journal of Marine Science and Application, 10(1):7, 2011. https://doi.org/10.1007/s11804-011-1035-8.

[25] M. Roseau. Contribution à la théorie des ondes liquides de gravité en profondeur variable, volume 275 . Service de documentation et d'information technique de l'aéronautique, 1952.

[26] D.J. Staziker, D. Porter and D.S.G. Stirling. The scattering of surface waves by local bed elevations. Applied Ocean Research, 18(5):283-291, 1996. https://doi.org/10.1016/S0141-1187(96)00021-1.

\section{Appendix}

Determination of the expression of $\phi_{0 x}(x, h)$.

We first construct a Green's function $g_{1}(\eta, y)$ corresponding to the equation (3.3). Here $g_{1}(\eta, y)$ satisfies

$$
\frac{\mathrm{d}^{4} g_{1}(\eta, y)}{\mathrm{d} \eta^{4}}-\gamma^{4} g_{1}(\eta, y)=\delta(\eta-y), \quad l_{1}<y, \eta<l_{2} ; \gamma^{4}=\frac{m K}{D}
$$

together with the end conditions

$$
g_{1}=0=g_{1 \eta}, \quad \eta=l_{1}, \quad g_{1 \eta \eta}=0=g_{1 \eta \eta \eta}, \quad \eta=l_{2} .
$$

Other inherent properties satisfied by $g_{1}$ :

$$
\left.\begin{array}{l}
g_{1}, g_{1 \eta}, g_{1 \eta \eta} \text { to be continuous at } \eta=y, \\
\left(\frac{\partial^{3} g_{1}}{\partial \eta^{3}}\right)_{\eta=y+0}-\left(\frac{\partial^{3} g_{1}}{\partial \eta^{3}}\right)_{\eta=y-0}=-1 .
\end{array}\right\}
$$

The general solution of equation (A.1) is given by

$$
g(\eta, y)=\left\{\begin{array}{l}
\sum_{i=1}^{4} A_{i}(y) e^{\gamma_{i} \eta}, l_{1}<\eta<y<l_{2}, \\
\sum_{i=1}^{4} B_{i}(y) e^{\gamma_{i} \eta}, l_{1}<y<\eta<l_{2},
\end{array}\right.
$$


where $\gamma_{1}, \gamma_{2}= \pm \mathrm{i} \gamma, \gamma_{3}, \gamma_{4}= \pm \gamma$ and the unknowns $A_{i}$ and $B_{i}$ 's are found by solving a matrix equation $\mathcal{P} \mathcal{Q}=\mathcal{R}$, where

$$
\mathcal{P}=\left[\begin{array}{cccc}
\mathrm{e}^{-\mathrm{i} \gamma l_{1}} & \mathrm{e}^{\mathrm{i} \gamma l_{1}} & \mathrm{e}^{-\gamma l_{1}} & \mathrm{e}^{\gamma l_{1}} \\
\mathrm{ie}^{-\mathrm{i} \gamma l_{1}} & -\mathrm{i} \mathrm{e}^{\mathrm{i} \gamma l_{1}} & \mathrm{e}^{-\gamma l_{1}} & -\mathrm{e}^{\gamma l_{1}} \\
-\mathrm{e}^{\mathrm{i} \gamma l_{2}} & -\mathrm{e}^{-\mathrm{i} \gamma l_{2}} & \mathrm{e}^{\gamma l_{2}} & \mathrm{e}^{-\gamma l_{2}} \\
-\mathrm{i} \mathrm{e}^{\mathrm{i} \gamma l_{2}} & \mathrm{ie}^{-\mathrm{i} \gamma l_{2}} & \mathrm{e}^{\gamma l_{2}} & -\mathrm{e}^{-\gamma l_{2}}
\end{array}\right], \quad \mathcal{Q}=\left[\begin{array}{c}
A_{1} \\
A_{2} \\
A_{3} \\
A_{4}
\end{array}\right]
$$

and $\mathcal{R}=\frac{1}{4 \gamma^{3}}\left[\begin{array}{c}0 \\ 0 \\ -\mathrm{i} \mathrm{e}^{\mathrm{i} \gamma\left(l_{2}-y\right)}+\mathrm{ie}^{-\mathrm{i} \gamma\left(l_{2}-y\right)}+\mathrm{e}^{\gamma\left(l_{2}-y\right)}-\mathrm{e}^{-\gamma\left(l_{2}-y\right)} \\ \mathrm{e}^{\mathrm{i} \gamma\left(l_{2}-y\right)}+\mathrm{e}^{-\mathrm{i} \gamma\left(l_{2}-y\right)}+\mathrm{e}^{\gamma\left(l_{2}-y\right)}+\mathrm{e}^{-\gamma\left(l_{2}-y\right)}\end{array}\right]$, together with the relations

$$
B_{1}=A_{1}-\frac{\mathrm{i}}{4 \gamma^{3}} \mathrm{e}^{-\mathrm{i} \gamma y}, B_{2}=A_{2}+\frac{\mathrm{i}}{4 \gamma^{3}} \mathrm{e}^{\mathrm{i} \gamma y}, B_{3}=A_{3}-\frac{1}{4 \gamma^{3}} \mathrm{e}^{-\gamma y}, B_{4}=A_{4}+\frac{1}{4 \gamma^{3}} \mathrm{e}^{\gamma y} .
$$

Now the solution of the equation (3.3) is expressed as

$$
\phi_{0 x}(0, y)=\frac{\gamma^{4}}{m} \int_{l_{1}}^{l_{2}} g_{1}(\eta, y)\left(\phi_{01}(0, \eta)-\phi_{02}(0, \eta)\right) \mathrm{d} \eta, \text { for } l_{1}<y<l_{2} \text {, }
$$

where $\phi_{01} \equiv \phi_{0}^{-}$and $\phi_{02} \equiv \phi_{0}^{+}$. Note that the above expression is not enough for numerical computation of $\phi_{0 x}$ as its right hand side involves the unknown potential difference function $\left(\phi_{01}(\eta)-\phi_{02}(\eta)\right)$.

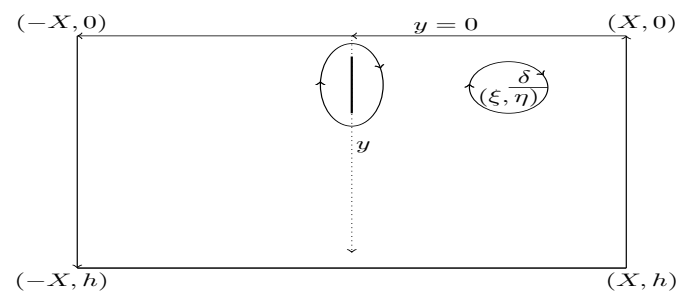

Figure 13. Contour for application of Green's integral theorem.

We thus seek for an alternative expression of $\phi_{0 x}(0, y)$. Applying Green's integral theorem to $\phi_{0 x}(0, y)-\mathrm{e}^{\mathrm{i} k_{0} x} \psi_{0}(y)$ and $\mathcal{G}(x, y ; \xi, \eta)$ (the fundamental potential function due to line source at $(\xi, \eta))$ in the domain given in Figure 13 we obtain an integral representation of $\phi_{0}(x, y)$ as (cf. Chakraborty et al. [2])

$$
\phi_{0}(x, y)=\mathrm{e}^{\mathrm{i} k_{0} x} \psi_{0}(y)-\int_{l_{1}}^{l_{2}}\left(\phi_{01}(0, \eta)-\phi_{02}(0, \eta)\right) \frac{\partial \mathcal{G}}{\partial \xi}(0, \eta ; x, y) \mathrm{d} \eta .
$$

Differentiating both sides with respect to $x$ and taking the limit $x \rightarrow 0$ we get

$$
\phi_{0 x}(0, y)=\frac{\partial}{\partial x}\left[\mathrm{e}^{\mathrm{i} k_{0} x} \psi_{0}(y)\right]_{x=0}-f_{l_{1}}^{l_{2}}\left[\phi_{01}-\phi_{02}\right](0, \eta) \frac{\partial^{2} \mathcal{G}}{\partial x \partial \xi}(0, \eta ; 0, y) \mathrm{d} \eta .
$$

The integration is performed in the sense of Hadamard finite-part integral and thus a cross is put on the integral sign. The two expressions of $\phi_{0 x}$ as given in 
the equations (A.2) and (A.4) are compared to yield the following hypersingular integral equation

$$
f_{l_{1}}^{l_{2}}\left[\frac{\partial^{2} \mathcal{G}}{\partial x \partial \xi}(0, \eta ; 0, y)+\frac{\gamma^{4}}{m} g_{1}(\eta, y)\right]\left[\phi_{01}-\phi_{02}\right](0, \eta) \mathrm{d} \eta=\frac{\partial}{\partial x}\left[\mathrm{e}^{\mathrm{i} k_{0} x} \psi_{0}(y)\right]_{x=0} .
$$

This technique is legitimated by several researchers (cf. Gayen and Mondal [8]) for studying the problems of waves scattering by thin plates. Considering the transformation $(y, \eta)=\frac{l_{2}-l_{1}}{2}+\frac{l_{2}-l_{1}}{2}(u, t)$ we can rewrite equation (A.5) as

$$
f_{-1}^{1} f(t)\left[-\frac{1}{(u-t)^{2}}-\kappa(u, t)\right] \mathrm{d} t=\left(l_{2}-l_{1}\right) \pi \mathrm{i} k_{0} \psi_{0}(u), \quad-1<u<1,
$$

where $f(t)=\left[\phi_{01}-\phi_{02}\right](0, t)$ and

$$
\begin{aligned}
& \kappa(u, t)=\left(\frac{l_{2}-l_{1}}{2}\right)^{2}\left[-\frac{1}{Y^{2}}+2 \int_{0}^{\infty} \frac{k \mathrm{e}^{-k h} \sinh k \eta \sinh k y}{\cosh k h} \mathrm{~d} k-2 \pi \frac{\gamma^{4}}{m} g_{1}(\eta, y)\right. \\
& \left.+2 \pi \mathrm{i}\left\{\frac{2 k_{0}^{2} \cosh k_{0}(h-\eta) \cosh k_{0}(h-y)}{2 k_{0} h+\sinh 2 k_{0} h}+\sum_{n=1}^{\infty} \frac{2 k_{n}^{2} \cos k_{n}(h-\eta) \cos k_{n}(h-y)}{2 k_{n} h+\sin 2 k_{n} h}\right\}\right] .
\end{aligned}
$$

In the above expression of $\kappa(u, t), Y=y+\eta$ and $k_{n}(n=1,2, \ldots)$ are positive real roots of $K \cos k h+k \sin k h=0$. The unknown function $f(t)$ is expanded as

$$
f(t)=\left(1-t^{2}\right)^{1 / 2} \sum_{n=0}^{N} a_{n} U_{n}(t) .
$$

The above approximation for $f(t)$ is consistent with the end point behaviour (2.4) of $\phi$. Here $a_{n}(n=0,1,2 \ldots, N)$ are the unknown constants to be determined and $U_{n}$ are the Chebyshev polynomials of the second kind. Substituting this expression of $f(t)$ in equation (A.6) and collocating at $(N+1)$ points $u=u_{j}=\cos \frac{2 j+1}{2 N+2}, j=0,1,2, \ldots, N$ we determine a system of $(N+1)$ linear equations, given by

$$
\begin{aligned}
& \sum_{n=0}^{N} a_{n} P_{n}\left(u_{j}\right)=\left(l_{2}-l_{1}\right) \pi \mathrm{i} k_{0} \psi_{0}\left(u_{j}\right), \\
& P_{n}\left(u_{j}\right)=\pi(n+1) U_{n}\left(u_{j}\right)+\int_{-1}^{1}\left(1-t^{2}\right)^{1 / 2} \kappa\left(u_{j}, t\right) U_{n}(t) \mathrm{d} t .
\end{aligned}
$$

Solving the system of equations (A.8) for $a_{n}$ 's and substituting them into equation (A.7) we get the estimation for $f(t)$. Also, from equation (A.3) we obtain

$$
\phi_{0 x}(x, h)=\mathrm{i} k_{0} \mathrm{e}^{\mathrm{i} k_{0} x} \psi_{0}(h)-\int_{l_{1}}^{l_{2}}\left(\phi_{01}(0, \eta)-\phi_{02}(0, \eta)\right) \frac{\partial^{2} \mathcal{G}}{\partial x \partial \xi}(0, \eta ; x, h) \mathrm{d} \eta .
$$

Now, using the value of $f(t)\left(=\left[\phi_{01}-\phi_{02}\right](0, t)\right)$ which is already computed from equation (A.7) after solving the system (A.8), we determine $\phi_{0 x}(x, h)$. In a similar manner we express $\phi_{0 x}(-x, h)$. Using expressions of $\phi_{0 x}(x, h)$ and $\phi_{0 x}(-x, h)$ we obtain the value of $R_{1}$ from the equation (3.6). 